\title{
Discrete spectrum Analysis using Laplace transform and Volterra equations (DALV-method)
}

\author{
V. V. Kuidin, V. V. Zalipaev, D. R. Gulevich \\ ITMO University, St. Petersburg 197101, Russia \\ vladislav.kujdin@metalab.ifmo.ru
}

PACS 02.70.Hm, 02.30.Hq, 03.65.Ge

DOI 10.17586/2220-8054-2021-12-1-15-21

\begin{abstract}
The theory of excitons in two dimensional materials including graphene and transition metal dichalcogenides (TMD) is complicated, as there appears a screened interaction in equations. Such interaction can be represented as Keldysh potential. The exact solution does not seem to exist yet. The method of searching appropriate solutions to equations of quantum mechanics is believed to solve this problem by using Laplace transform of tempered distributions and Volterra equations. The method is to seek solution as a Laplace transform of some tempered distribution that satisfies the appropriate Laplace spectral equation which, under Laplace transform, gives us the initial equation. Due to Paly-Wigner-Schwarz theorem, the image functions behavior depends on the geometry of original one support. In addition, the homogenous Volterra equation does not have nontrivial continuous solution. These constraints together with the fact that the studied equations turn out to be Volterra equations of III kind lead to a method that seems to solve a wide class of quantum mechanics equations.
\end{abstract}

Keywords: discrete spectrum, Laplace transform, Volterra equation, Schrödinger equation.

Received: 10 January 2021

Revised: 3 February 2021, 12 February 2021

\section{Introduction}

In the past years, the spectral theory of differential operators has attained extensive results for various differential operators. The qualitative theory for second-order differential equations has been systematically presented e.g. in [1]. The results on spectral theory of differential equations has been presented in $[2,3]$. The asymptotic analysis has been developed for calculating spectrum of quantum mechanics operators [4].

However, there are few methods for exact calculation of discrete spectrum even for the most studied Schrödinger equation that can be utilized by physicists. The most used methods remain perturbation theory, semiclassical analysis and computational methods. Thus, the computationally simple method for computing discrete spectrum of quantum mechanics operators is of great necessity. One of the best rigorous approaches to calculating discrete spectrum widely used is Frobenius method, which is used to calculate the discrete spectrum and corresponding wave functions of an electron in hydrogen atom and constant magnetic and electric fields. Nevertheless, the Frobenius method fails when a non-fuchsian potentials (Keldysh potential), i.e. the potentials with non-fuchsian singularities, are studied.

Classical Laplace transform has been used to calculate discrete spectrum of Schrödinger equation with particular radially symmetric potentials. The original second-order differential equation is transformed to another differential or difference equation [5-11]. The fractional differential equations has been studied e.g. in [12]. The study of Schrödinger equation with Coulomb potential and a pseudoharmonic potential using the Laplace transform has been elaborated in $[6,10]$. The one-dimensional equation with periodic potentials having finite number of Fourier coefficients has been studied in [13]. The above papers utilized classical Laplace transform to reduce the original spectral problem to a more treatable one. However, most of studied equations can be reduced to Fuchsian ones by an appropriate substitution.

The main concept in DALV-method presented in this paper is to treat the solutions to Schrödinger equation as the Laplace transform of some generalized functions, whose properties have been systematically studied in [14]. In this paper, we attempt to derive a method to analyze the Schrödinger equation with a wide class of radially symmetric potentials. On the contrary to above papers, DALV-method utilizes the inverse Laplace transform to convert the original differential equation to convolutional equation with the solution being a generalized function.

The Laplace transform of a generalized function with certain properties of the support exhibits the required asymptotic behaviour (Theorem (3.10.2) [14]), which is the main starting point for presented method. A distribution whose Laplace transform is a function $f$ will be called Laplace spectral function of $f$. The equation for the Laplace spectral function of a solution to given differential equation will be denoted as Laplace spectral equation. The latter turns out to be a homogenous III kind Volterra equation, whose spectral properties has been studied in [15-17]. The 
coefficients of the initial equation need to have smooth Laplace spectral functions (up to a factor $\theta(s)$ ) for the corresponding Laplace spectral equation to become a Volterra equation with smooth kernel. Some potentials with smooth Laplace spectral functions are given in Table 1. The homogenous Volterra equation's solution properties along with the behaviour of weight function lead to the choice of a particular ansatz, which, after being applied to the spectral equation, results in the system of algebraic and integral equations for ansatz elements. The singular points of the solution corresponds with the eigenvalue, so the spectrum can be calculated without calculating the eigenfunction. As a result, the formula for energy levels is obtained and the equations for corresponding eigenfunctions are presented.

\section{Preliminaries}

\subsection{Laplace transform of generalized functions}

According to Zemanian [14], we will introduce Laplace transform of a regular compactly supported generalized function as follows:

$$
\mathcal{L}_{s \rightarrow r} F \stackrel{\text { def }}{=}\left(F, e^{-r s}\right)=\int_{\mathbb{R}} F(s) e^{-r s} \mathrm{~d} s .
$$

The definition can be expanded to tempered distributions supported on $\mathbb{R}_{+}$. Throughout the whole paper, we will utilize tempered distributions with semibounded supports, namely $\operatorname{supp}(f) \subset \mathbb{R}_{+}$. The above definition is valid for such distributions, as for every $r>0$ the function $\eta(s) e^{-r s}, \eta \in C^{\infty}(\mathbb{R}), \eta([-1, \infty))=1, \operatorname{supp}(\eta) \subset[-T,+\infty)$ for some $T \geqslant 2$ is in Schwartz class, so the definition (1) is correct as

$$
\left(F, e^{-r s}\right)=\left(F, \eta(s) e^{-r s}\right) .
$$

The definition does not depend on $\eta$ when we apply a semibounded distribution to it since $\partial_{n} \eta(s) e^{-r s}=\partial_{n} e^{-r s}$, $s \geqslant 0, n=0,1,2 \ldots$. In fact, $\eta$ is added rather for technical reasons so that the tempered distribution acts on the function from Schwartz class as the theory of tempered distributions demands it.

Throughout the whole paper, we will utilize tempered distributions with semibounded supports, namely $\operatorname{supp}(f) \subset \mathbb{R}_{+}$.

All well-known properties of Laplace transform can be proved for Zemanian's definition:

$$
\begin{gathered}
\mathcal{L}_{s \rightarrow r} F^{(n)}(s)=r^{n}\left(\mathcal{L}_{s \rightarrow r} F\right)(r), n=0,1,2 \ldots, \\
\mathcal{L}_{s \rightarrow r} s^{n} F(s)=(-1)^{n}\left(\mathcal{L}_{s \rightarrow r} F\right)^{(n)}(r), n=0,1,2 \ldots, \\
\mathcal{L}_{s \rightarrow r} F(s-a)=e^{-a r}\left(\mathcal{L}_{s \rightarrow r} F\right)(r), a \in \mathbb{R} .
\end{gathered}
$$

Another property of Laplace transform to be used in this paper is the Laplace transform of a convolution:

$$
\mathcal{L}_{s \rightarrow r}(F * G)=\mathcal{L}_{s \rightarrow r} F \cdot \mathcal{L}_{s \rightarrow r} G .
$$

\subsection{Laplace spectral function and the integrability condition}

The main concept of DALV-method is to consider the solution to Schrödinger equation as the Laplace transform of some tempered distribution. The equation for this distribution will be called Laplace spectral equation and the distribution whose Laplace transform is some function $f$ will be called Laplace spectral function of $f$. Unlike Fourier transform, Laplace transform is not an isometry on $L_{2}(\mathbb{R})$. Therefore, the integrability of the wave-function should somehow be interpreted in terms of its Laplace spectral function.

When treating the Schrödinger equation in $\mathbb{R}^{n}$, the radial part of wavefunction should satisfy the integrability condition:

$$
\int_{0}^{\infty} r^{n-1}|R(r)|^{2} \mathrm{~d} r<\infty
$$

First assume that $R(r)=\left(F, e^{-r s}\right)$ and $F$ is a smooth compactly supported function. Observe that an integral:

$$
\int_{0}^{\infty} r^{n-1} e^{-r s} \mathrm{~d} r
$$

exists only when $s>0$. In this case, we can change the order of integration and the integral in (3) can be rewritten as:

$$
\int_{0}^{\infty} r^{n-1}|R(r)|^{2} \mathrm{~d} r=\Gamma(n) \int_{\mathbb{R}_{+}} \frac{F(s) * \overline{F(s)}}{s^{n}} \mathrm{~d} s, \operatorname{supp}(F) \subset(0,+\infty) .
$$


TABLE 1. Various potentials and their Laplace spectral functions. $\theta(s)$ - Heaviside theta function, $J_{0}(r), Y_{0}(r)$ - Bessel functions of first and second type respectively, $H_{0}(r)$ - Struve function

\begin{tabular}{|l|c|c|}
\hline & Potential & Spectral function \\
\hline Coulomb potential & $V(r)=\frac{1}{r}$ & $K(s)=\theta(s)$ \\
\hline Shifted Coulomb potential & $V(r)=\frac{1}{\sqrt{r^{2}+a^{2}}}$ & $K(s)=\theta(s) J_{0}(a s)$ \\
\hline Keldysh potential & $V(r)=\frac{\pi}{2}\left(H_{0}(r)-Y_{0}(r)\right)$ & $K(s)=\frac{\theta(s)}{\sqrt{1+s^{2}}}$ \\
\hline “Centripedal potential” & $V(r)=\frac{1}{r^{2}}$ & $K(s)=\theta(s) s=s_{+}$ \\
\hline
\end{tabular}

This gives us the integrability condition for a function with smooth compactly supported Laplace spectral function:

$$
\int_{\mathbb{R}_{+}} \frac{F(s) * \overline{F(s)}}{s^{n}} \mathrm{~d} s<\infty .
$$

A few crucial conclusions can be made from this result. First, the support of $F$ can only lie on the right semiaxis $\mathbb{R}_{+}$. Second, the function $F$ has to vanish in the neighborgood of zero. A similar result is obtained for tempered distributions, but with certain technical subtleties.

Since $R(r)=\left(F, e^{-r s}\right), \operatorname{supp}(F) \subset \mathbb{R}_{+}$, we can rewrite (3) using the notion of generalized functions:

$$
\int_{0}^{\infty}\left(F * \bar{F}, r^{n-1} e^{-r s}\right) \mathrm{d} r<\infty
$$

Observe now the integral

$$
\int_{a}^{\infty} r^{n-1} e^{-r s} \mathrm{~d} r=\frac{\Gamma(n, a s)}{s^{n}}, s>0,
$$

where $\Gamma(n, a s)$ is an incomplete gamma function. Again, since $\eta \Gamma(n, a s)$ with the above mentioned $\eta$ is from Schwartz space and $\operatorname{supp}(F * \bar{F}) \subset \mathbb{R}_{+}$if $\operatorname{supp}(F) \subset \mathbb{R}_{+}$, we can formulate the condition (3) in terms of spectral functions:

$$
\lim _{a \rightarrow 0}\left(\frac{F * \bar{F}}{s^{n}}, \Gamma(n, a s)\right)<\infty .
$$

In terms of Laplace transformable generalized functions [14], the above inequality means that the Laplace transform of a distribution $s^{-n}(F * \bar{F})$ is defined at point zero (clearly, $\Gamma(n, 0)=\Gamma(n)=$ const).

\section{Laplace spectral equation, associated with Schrödinger equation}

The main equation studied in this paper is the radial part of the Schrödinger equation with radially symmetric potential $V(r)$ having a smooth Laplace spectral function. This equation is reduced to one-dimensional equation:

$$
-\frac{1}{2} f^{\prime \prime}(r)+\left(V(r)+\frac{l(l+1)}{2 r^{2}}\right) f(r)=E f(r), r>0
$$

The corresponding Laplace spectral equation, assuming that

$$
2 V(r)+\frac{l(l+1)}{r^{2}}=\int_{0}^{\infty} e^{-r s}(2 K(s)+l(l+1) s) \mathrm{d} s=\int_{0}^{\infty} e^{-r s} K_{e f f}(s) \mathrm{d} s
$$

with a smooth function $K(s)$ and $F: f(r)=\mathcal{L}_{s \rightarrow r} F$ being the Laplace spectral function of $f$ reads:

$$
\theta(s) K_{e f f}(s) * \theta(s) F(s)=\left(s^{2}-s_{0}^{2}\right) F(s) .
$$


Where $s_{0},-s_{0}^{2}=2 E$, is a spectral parameter. It is well known that the discrete spectrum is to be sought in $\mathbb{R}_{-}$, so $s_{0}$ is to be sought in $\mathbb{R}$. The function $\left(s^{2}-s_{0}^{2}\right)$ has two zeros $\pm s_{0}$, but $\operatorname{supp}(F) \subset \mathbb{R}_{+}$, so we assume further that $s_{0} \geqslant 0$. The factor $\theta(s)$ before $F(s)$ is added for symmetry. In order to justify the equation (9), one can apply Laplace transform to it:

$$
\begin{gathered}
\mathcal{L}_{s \rightarrow r} \theta(s) K_{\text {eff }}(s) * \theta(s) F(s)=\left(2 V(r)+\frac{l(l+1)}{r^{2}}\right) \mathcal{L}_{s \rightarrow r} F, \\
\mathcal{L}_{s \rightarrow r}\left(\left(s^{2}-s_{0}^{2}\right) F(s)\right)=\left(\mathcal{L}_{s \rightarrow r} F\right)^{\prime \prime}+2 E \mathcal{L}_{s \rightarrow r} F .
\end{gathered}
$$

Rearranging the terms, we end up with the original equation (8).

The form of the equation assumes the solution to have singularities at least at point $s=s_{0}$. Moreover, the regular part of solution has to be zero in the interval $\left(0, s_{0}\right)$, since for $s \in\left(0, s_{0}\right)$ the equation can be reduced to homogenous Volterra one with regular kernel. The support of the Laplace spectral function being separated from 0 gives us the appropriate behaviour of the wavefunction in the neighborgood of infinity. The only problem to be resolved would then be the behaviour of Laplace spectral function in the neighborgood of infinity, that correlates with the behaviour of wavefunction near 0 .

The singularity at point $s=s_{0}$ is believed to be accounted for by a set of some specific generalized functions. However, this set does not necessarily account for the regular part of solution. Therefore, the solution shall be constructed in view of the above. The ansatz for the solution can be chosen the following:

$$
\begin{gathered}
F(s)=\theta\left(s-s_{0}\right) \chi\left(s-s_{0}\right)+\sum_{n=-\lceil\gamma\rceil}^{N} A_{n} \varphi_{-\gamma-n}\left(s-s_{0}\right), \quad \gamma>0, N \in \mathbb{N}, \\
\varphi_{a}(s)= \begin{cases}\frac{\theta(s) s^{a-1}}{\Gamma(a)}, & a>0, \\
\varphi_{a+N}^{(N)}, & a<0, a+N>0, N \in \mathbb{N} .\end{cases}
\end{gathered}
$$

Where $\varphi_{a+N}^{(N)}$ is a generalized derivative of $\varphi_{a+N}$. Here, $\chi$ is a continuous function and the sum characterizes the singularity of solution. The sum is finite since we seek solution in the set of tempered distributions having finite order. The functions $\varphi_{a}(s)$ are the kernels of a fractional Riemann-Liouville derivatives. Their main properties are shown in [18] and their Laplace transforms are

$$
\mathcal{L}_{s \rightarrow r} \varphi_{-\gamma-n}\left(s-s_{0}\right)=e^{-s_{0} r} r^{\gamma+n} .
$$

Note that $\varphi_{a}(s)$ has singularity at $s=0$, whenever $a<1$. Therefore, we attempt to extract singularities of a spectral function by applying such ansatz. The possibility to separate singular part from regular one by proposed linear combination remains an open problem for now. Although, in some cases, such ansatz might give reliable values for spectrum and corresponding eigenfunctions. However, in order to account for all singularities at point $s=s_{0}$, the more generic representation of a singular part might be needed:

$$
F_{\text {sing }}(s)=\int_{0}^{N} A(\alpha) \varphi_{-\alpha+1}\left(s-s_{0}\right) \mathrm{d} \alpha .
$$

The algebraic iteration formulae would then become difference equation for $A(\alpha)$. In this paper, we utilize the ansatz (12) to show, that it gives an exact solution for Coulomb interaction.

\section{Singular and regular parts}

When convolving $\theta(s) K(s)$ with $\varphi_{-\gamma-n}\left(s-s_{0}\right)=\varphi_{1-\{\gamma\}}^{(\lceil\gamma\rceil+n)}\left(s-s_{0}\right)$, where $\lceil\gamma\rceil-$ ceiling function and $\{\gamma\}=$ $\gamma-\lfloor\gamma\rfloor$, we get:

$$
\theta(s) K(s) * \varphi_{1-\{\gamma\}}^{(\lceil\gamma\rceil+n)}\left(s-s_{0}\right)=(\theta(s) K(s))^{(\lceil\gamma\rceil+n)} * \varphi_{1-\{\gamma\}}\left(s-s_{0}\right) .
$$

Differentiation of $\theta(s) K(s)$ results in the linear combination of delta functions. The following formula:

$$
(\theta(s) K(s))^{(\lceil\gamma\rceil+n)}=\theta(s) K^{(\lceil\gamma\rceil+n)}(s)+\sum_{k=1}^{\lceil\gamma\rceil+n} C_{\lceil\gamma\rceil+n}^{k} \delta^{(k-1)}(s) K^{(\lceil\gamma\rceil+n-k)}(s),
$$

where $C_{\lceil\gamma\rceil+n}^{k}$ is a binomial coefficient, gives us regular and singular terms. The former becomes the nonhomogenity for a regular integral equation, the latter gives coefficients for an algebraic system. The expression $\delta^{k-1}(s) K^{(\lceil\gamma\rceil+n-k)}(s)$ needs to be calculated as a multiplication of Dirac delta function and a smooth function. After applying the ansatz (12) to equation (9), the latter splits into two parts: singular and regular ones. The singular 
part could be resolved into the iterative system of equations for $A_{n}$, whereas the regular one gives us the equation for $\chi$. After some algebraic manipulations, we obtain the system

$$
\begin{aligned}
& \sum_{j=n-\lceil\gamma\rceil+1}^{N} A_{j} K_{e f f}^{(j+\lceil\gamma\rceil-n-1)}(0)= \\
& \quad=(\gamma-\lceil\gamma\rceil+n+2)(\gamma-\lceil\gamma\rceil+n+1) A_{n-\lceil\gamma\rceil+2}-2 s_{0}(\gamma-\lceil\gamma\rceil+n+1) A_{n-\lceil\gamma\rceil+1},
\end{aligned}
$$

for $n=0, \ldots, N+\lceil\gamma\rceil-1$. The requirement $A_{N} \neq 0$, and the requirement $A_{N+k}=0, k \in \mathbb{N}$, gives us the formula for $s_{0}$ :

$$
s_{0}=-\frac{K_{e f f}(0)}{2(N+\gamma)} \Leftrightarrow E_{N}=-\frac{K_{e f f}^{2}(0)}{8(N+\gamma)^{2}} .
$$

The search of $\gamma$ can be described the following: the iterative system (16) has to zero out at some point. The possibility for $\gamma$ to have multiple solutions (not exactly positive), or no solutions at all, requires further study.

The equation (16) gives us the formulae for coefficients:

$$
A_{n-1}=\frac{\gamma+N}{K_{e f f}(0)(N+m)}\left(\left((\gamma+n+1)(\gamma+n)-K_{e f f}^{\prime}(0)\right) A_{n}-\sum_{j=n+1}^{N} A_{j} K_{e f f}^{(j-n)}(0)\right)
$$

The coefficient $A_{N}$ is chosen arbitrarily. Since $A_{n}$ and $\chi(s)$ depend linearly on $A_{N}$, the whole solution is unique up to a constant factor. This makes $A_{N}$ the norming factor. The term $\chi(s)$ from formula (12) is found from the equation

$$
\int_{0}^{z} K(z-t) \chi(t) d t+G(z)=z\left(z+2 s_{0}\right) \chi(z), \quad z>0
$$

with $G(z)$ obtained from convolution of kernel with singular part of solution:

$$
G(z)=\sum_{n=-\lceil\gamma\rceil}^{N} \frac{A_{n}}{\Gamma(1-\{\gamma\})} \int_{0}^{z} \frac{d^{\lceil\gamma\rceil+n} K_{e f f}(t)}{d t^{\lceil\gamma\rceil+n}} \frac{\mathrm{d} t}{(z-t)^{\{\gamma\}}} .
$$

The equation (19) is a Volterra equation of III kind. The theory of integral equations of III kind has not been fully developed comparing to that for integral equations of I and II kinds. Fredholm equations of III kind has been studied, for example, in [15]. The authors showed that the solution of such equation has singularities at points, where the weight function vanishes. In the above case, the operator

$$
\mathcal{K} \varphi(z)=\int_{0}^{z} \frac{K_{e f f}(z-t)}{z\left(z+2 s_{0}\right)} \varphi(t) \mathrm{d} t=\int_{0}^{1} \frac{K_{e f f}(z(1-x))}{\left(z+2 s_{0}\right)} \varphi(z x) \mathrm{d} x
$$

belongs to the class of cordial operators, whose spectral properties has been systematically studied in [16,17]. According to those papers, the spectrum of operator (21) in continuous functions is $\sigma_{0}(\mathcal{K})=-(N+\gamma)(\lambda+1)^{-1}, \operatorname{Re} \lambda \geq 0$. Therefore, the equation (19) that can be written as

$$
\chi(z)=\mathcal{K} \chi(z)+\frac{G(z)}{z\left(z+2 s_{0}\right)}=\mathcal{K} \chi(z)+\hat{G}(z), \quad z>0
$$

has a unique continuous solution $\chi(z)$, once $\hat{G}$ is continuous at zero. However, if $\gamma \notin \mathbb{Z}$, the free term in (22) has a weak singularity. In this case, the theory in [17] is to be generalized for $L_{1}\left(\mathbb{R}_{+}\right)$space.

\section{Coulomb potential}

The spectral function of a coulomb potential $V(r)=\frac{Z}{r}$ is a constant $K(s)=Z$ (it actually is a Heaviside function, but the multiplication by one is already taken into consideration), therefore all its derivatives vanish and the formulae for $A_{n}$ contain only two adjacent coefficients. The system (16) zeroes out at coefficient $A_{-1}$ when $\gamma=l+1$, so the system (16) becomes an iteration formula

$$
A_{n}=\frac{(n+1)(2 l+n+2)}{-2 Z+2 s_{0}(l+n+1)} A_{n+1},
$$


which is exactly the formula for coefficients of a polynomial part of hydrogen atom wavefunction. The formula (17) becomes a well-known Rydberg formula

$$
s_{0}=\frac{Z}{(N+l+1)} .
$$

The regular equation (19) becomes trivial, as (20) equals to zero. Therefore, we obtain the eigenvalues and eigenfunctions:

$$
E_{N}=-\frac{Z^{2}}{2(N+l+1)^{2}}, \quad f_{N, l}(r)=r^{l+1} e^{-s_{0} r} \sum_{n=0}^{N} A_{n} r^{n} .
$$

\section{Generalizations}

The proper analysis of Schrödinger equation, however, has to be conducted explicitly without substitutions like $R(r)=r^{-1} f(r)$, so there would be no additional assumptions on Laplace spectral function behaviour. Then the Laplace spectral equation belongs to a wider class of equations:

$$
\sum_{\alpha, \beta} s^{\alpha}\left(\theta(s) K^{\alpha \beta}(s) * \theta(s) s^{\beta} F(s)\right)=a\left(s, s_{0}(E)\right) F(s) .
$$

In fact, a wide range of pseudodifferential equations belongs to this class, including Dirac-type equations (after reducing the latter to the scalar equation). Indeed, the operator

$$
A F=\sum_{\alpha, \beta} s^{\alpha}\left(\theta(s) K^{\alpha \beta}(s) * \theta(s) s^{\beta} F(s)\right),
$$

after Laplace transform, gives us the operator

$$
\mathcal{L}_{s \rightarrow r} A \psi=\sum_{\alpha, \beta} \frac{\partial^{\alpha}}{\partial r^{\alpha}} V^{\alpha \beta}(r) \frac{\partial^{\beta}}{\partial r^{\beta}} \psi(r) .
$$

This gives the opportunity to cover a wide class of spectral problems by means of DALV-method.

\section{Conclusion}

The approach to analyzing a spectral problem in real space by treating a solution as the Laplace transform of a tempered distribution is believed to give extensive results in quantitative analysis of discrete spectrum of differential operators. The study of Laplace spectral equations gives identical results for already solved equation for hydrogen atom. The discrete linear combination of Riemann-Liouville kernels can be replaced with continuous one, following the logic of Fourier transform theory evolution. Moreover, the equation (9) could be obtained for a wider class of differential and even pseudodifferential operators on $\mathbb{R}_{+}$. The analysis of a Dirac-type matrix differential operators with DALV-method gives a convincing formulae for spectrum that could result in the rise of a self-sustained theory. Nevertheless, the rigorous proofs are to be elaborated for the method to become purely mathematical.

\section{Acknowledgements}

The work is supported by the Russian Science Foundation under the Grant No. 18-12-00429.

\section{References}

[1] Titchmarsh E.G., Teichmann T. Eigenfunction expansions associated with secondorder differential equations, part 2. PhT, 1958, 11(10), P. 34.

[2] Teschl G. Mathematical Methods in Quantum Mechanics: With Applications to Schrödinger Operators. Graduate studies in mathematics. American Mathematical Society, 2009.

[3] Dunford N., Schwartz J.T. Linear Operators, Part 2: Spectral Theory, Self Adjoint Operators in Hilbert Space. Wiley Classics Library, Wiley, 1988.

[4] Fedoryuk M.V., Maslov V.P. Quasiclassical approximation for quantum mechanics equations, 1976.

[5] Englefield M.J. Solution of Schrödinger equation by Laplace transform. Journal of The Australian Mathematical Society, 1968, 8(3), P. 557567.

[6] Englefield M.J. Solution of coulomb problem by Laplace transform. Journal of Mathematical Analysis and Applications, 1974, 48(1), P. 270275.

[7] Altuüg Arda and Ramazan Sever. Exact solutions of the Schrödinger equation via Laplace transform approach: pseudoharmonic potential and mietype potentials. Journal of Mathematical Chemistry, 2012, 50(4), P. 971-980.

[8] Douglas R.M., Pimentel and Antonio S De Castro. A Laplace transform approach to the quantum harmonic oscillator. European Journal of Physics, 2012, 34(1), P. 199.

[9] Ginyih Tsaur and Jyhpyng Wang. A universal Laplace transform approach to solving Schrödinger equations for all known solvable models. European Journal of Physics, 2013, 35(1), P. 015006. 
[10] Tapas Das and Altüg Arda. Exact analytical solution of the ndimensional radial Schrödinger equation with pseudoharmonic potential via Laplace transform approach. Advances in High Energy Physics, 2015, 2015.

[11] Yangqiang Ran, Lihui Xue, Sizhu Hu, and RuKeng Su. On the coulombtype potential of the onedimensional Schrödinger equation. Journal of Physics A: Mathematical and General, 2000, 33(50), P. 9265.

[12] Naber M. Time fractional Schrödinger equation. Journal of Mathematical Physics, 2004, 45(8), P. 3339-3352.

[13] Michael E. Clarkson and Huw O Pritchard. A Laplace transform solution of Schrödingers equation using symbolic algebra. International journal of quantum chemistry, 1992, 41(6), P. 829-844.

[14] Zemanian A.H. Generalized integral transformations, 1968.

[15] Warnock R.L. Bart G.R. Linear integral equations of the third kind. SIAM Journal on Mathematical Analysis, 1973, 4(4), P. 609-622.

[16] Vainikko G. Cordial Volterra integral equations 1. Numerical Functional Analysis and Optimization, 2009, 30(910), P. 1145-1172.

[17] Vainikko G. Cordial Volterra integral equations 2. Numrical Functional Analysis and Optimization, 2010, 31(2), P. 191-219.

[18] Vladimirov V.S. Generalized functions in mathematical physics, Nauka, Moscow, 1979. English transl, Mir, Moscow, 1979. 